\title{
Judgment aggregation in search for the truth: the case of interconnections
}

Citation for published version (APA):

Bozbay, I. (2012). Judgment aggregation in search for the truth: the case of interconnections. METEOR, Maastricht University School of Business and Economics. METEOR Research Memorandum No. 027 https://doi.org/10.26481/umamet.2012027

Document status and date:

Published: 01/01/2012

DOI:

10.26481/umamet.2012027

Document Version:

Publisher's PDF, also known as Version of record

\section{Please check the document version of this publication:}

- A submitted manuscript is the version of the article upon submission and before peer-review. There can be important differences between the submitted version and the official published version of record.

People interested in the research are advised to contact the author for the final version of the publication, or visit the DOI to the publisher's website.

- The final author version and the galley proof are versions of the publication after peer review.

- The final published version features the final layout of the paper including the volume, issue and page numbers.

Link to publication

\footnotetext{
General rights rights.

- You may freely distribute the URL identifying the publication in the public portal. please follow below link for the End User Agreement:

www.umlib.nl/taverne-license

Take down policy

If you believe that this document breaches copyright please contact us at:

repository@maastrichtuniversity.nl

providing details and we will investigate your claim.
}

Copyright and moral rights for the publications made accessible in the public portal are retained by the authors and/or other copyright owners and it is a condition of accessing publications that users recognise and abide by the legal requirements associated with these

- Users may download and print one copy of any publication from the public portal for the purpose of private study or research.

- You may not further distribute the material or use it for any profit-making activity or commercial gain

If the publication is distributed under the terms of Article $25 \mathrm{fa}$ of the Dutch Copyright Act, indicated by the "Taverne" license above, 


\section{Maastricht University}

I rem Bozbay

J udgment aggregation in search for the truth: the case of interconnections

RM/ 12/027

\section{METEOR}

Maastricht University School of Business and Economics

Maastricht Research School of Economics

of Technology and Organization

P.O. Box 616

NL - 6200 MD Maastricht

The Netherlands 


\title{
Judgment aggregation in search for the truth: the case of interconnections
}

\author{
İrem Bozbay*
}

December 2011

\begin{abstract}
This paper analyses the problem of aggregating judgments when strategic voters hold private information about which propositions are true and share a common preference for true collective judgments. We go beyond previous work by introducing logical interconnections between the propositions. A voter's private information can be inconclusive. The goal is to determine the voting rules which lead to collective judgments that efficiently incorporate all private information. We characterize the (rare) situations in which such rules exist, as well as the nature of these rules.
\end{abstract}

\section{Introduction}

The theory of judgment aggregation deals with situations where a group needs to make a collective 'yes' or 'no' judgment on several issues on the basis of group members' judgments on these issues. Many decision making problems in real life involve multiple issues. The jury in a court trial might need to form judgments on whether the defendant has broken the contract, and whether the contract is legally valid. The city council might need to reach judgments on whether the $\mathrm{CO}_{2}$ level in the city is above the critical threshold and whether the chemical plant in the city should be closed down. In such problems, the issues on the group's agenda might be mutually interconnected, in that the judgment made on one issue might constrain the judgment on another issue. In the city commission example, the $\mathrm{CO}_{2}$ level being judged to be above the critical threshold might restrict the judgment on the second issue to 'yes'; i.e., lead to the closing of the chemical factory. Judgment aggregation models allow for the study of a wide range of realistic collective decision making problems.

When it comes to aggregate judgments in decision making bodies like juries or city councils, it seems natural to have epistemic concerns. Such problems are different than problems where individuals have conflicting aims. In the court trial example, the jury's problem is to find out two independent facts, whether the defendant has broken the contract and whether the contract is legally valid. It seems that the primary goal is reaching the truth in such problems. The epistemic approach in

*Maastricht University, E-mail: i.bozbay@maastrichtuniversity.nl 
judgment aggregation aims to reach true group judgments. According to the classical social-choice theoretic approach in judgment aggregation - where voters are taken to have conflicting aims - a good voting rule should be fair to jurors while according to the epistemic approach, a good voting rule should track the truth. Whether a voting rule is good in tracking the truth or not depends on questions such as whether the individuals' judgments are sufficient evidence for the truth value of each issue, and whether individuals have expressed their judgments truthfully.

This chapter assumes that the group faces two issues, and a 'no' answer to both issues is inconsistent. Consistency is a property that requires the collective decision to be free from any logical contradictions. In the city council example, the inconsistency arises in case of a 'yes' judgment on the first issue $\left(\mathrm{CO}_{2}\right.$ level is above the threshold) and a 'no' judgment on the second issue (the chemical plant should not be closed down). We assume that voters share the common goal of tracking the truth but each has private information regarding the truth value of each issue. In this setting we want to answer the following question: Which voting rules lead to efficient and truthful Bayesian Nash equilibrium of the corresponding game? So, we want to design voting rules which first lead to truthful revelation of private information, and second lead to the efficiency in equilibrium. Note that individual reporting of private information need not always be truthful, even when voters have no conflicting aims. As AustenSmith and Banks (1996) show, if a voter conditions her beliefs on being pivotal on being able to change the outcome - she may not always find it best to report truthfully. The question of consistency here arises when one wants to use quota rules (where separate votes are taken on each proposition using acceptance thresholds) which are practical and common. This paper also examines the possibility of truthtracking in particular with quota rules.

The epistemic perspective with strategic concerns is well-established in a different body of the literature, which studies single issue problems (Austen-Smith and Banks 1996, Feddersen and Pesendorfer 1997, 1998). There have been few works taking the epistemic approach in judgment aggregation. The work by Bozbay, Dietrich and Peters (2011) studies judgment aggregation from the epistemic and strategic voting perspective. They model voters' common interests and private information, with an agenda with independent issues and they study the problem of designing a voting rule. We extend their work to agendas where issues are interconnected. Ahn and Oliveros (2011) and De Clippel and Eliaz (2011) study elections on multiple issues with common preferences and asymmetric information. Each of these papers compares two voting rules in terms of whether they efficiently aggregate information, asymptotically as the group size tends to infinity.

A series of possibility and impossibility results from the perspective of classical social-choice theoretic approach are successfully obtained in the judgment aggregation literature (e.g., List and Pettit 2002, Dietrich 2006, 2007, 2010, Nehring and Puppe 2008, 2010, Dietrich and List 2007a, 2007c, 2008, Dokow and Holzman 2010a, 2010b, Dietrich and Mongin 2010; see also precursor results by Guilbaud 1952 and Wilson 1975; for an introductory overview see List and Polak 2010). Ahn and Oliveros (2012) study voting games where voters hold private values of, but common information about issues. Few papers about judgment aggregation take a truth-tracking 
perspective without considering private information and strategic incentives (e.g., Bovens and Rabinowicz 2006 and List 2005). Dietrich and List (2007b) analyse strategic voting behaviour resulting from private values instead of private information. See also related work by Nehring and Puppe (2002, 2007). List and Pettit (2011) provide a systematic philosophical analysis of the truth-tracking approach.

This paper also relates to the body of work on binary choice problems where strategic voters with common interests and private information are considered. AustenSmith and Banks (1996) and Feddersen and Pesendorfer (1997) show that it typically cannot be rational for all voters to vote sincerely, and that the choice of voting rule matters considerably for sincere voting and efficient information aggregation. AustenSmith and Feddersen $(2005,2006)$ add an extra dimension of pre-voting deliberation. Duggan and Martinelli (2001) extend the approach to continuous rather than binary private information. Feddersen and Pesendorfer (1998), Coughlan (2000) and Gerardi (2000) examine the (in)effectiveness of unanimity rule in 'protecting the innocent' in jury trials.

The paper proceeds as follows. Two questions are answered in Section 2 and Section 3; respectively, when informative voting is efficient, and when informative voting is efficient by quota rules in particular. By informative voting, we generally mean 'following the evidence' whenever the evidence is consistent with the true state. ${ }^{1}$ Section 2.1 presents the model, in which a group of voters is to decide whether to accept or reject each of two propositions while they can not reject both at the same time. The model presented in Section 3.1 differs from the model in Section 2.1 in that the collective decision might be inconsistent; hence, both propositions can be collectively rejected. In both models, voters hold truth-tracking preferences and they vote on the basis of private information which may possibly be inconsistent. Section 2.2, 2.3 and 2.4 address the key question of how to design the voting rule such that it leads to efficient decisions as well as simple-minded, truthful voting behaviour in equilibrium. The answer depends on both the kind of utility function in use and the definition of simple-minded behaviour. It turns out that in many situations such a voting rule doesn't exist. The necessary and sufficient conditions for the existence of such rules are also given and these rules are characterized by some properties. Section 3.2 addresses the possibility of efficient information aggregation with quota rules and provides an impossibility result. All proofs are in appendix.

\section{Efficient information aggregation}

\subsection{The Model}

\subsubsection{A simple judgment aggregation problem}

We consider a group of voters, labeled $i=1, \ldots, n$, where $n \geq 2$. There are two propositions $p$ and $q$, and their negations $\bar{p}$ and $\bar{q}$. The group of voters wants to obtain a collective judgment on whether $p$ or $\bar{p}$ is true, and whether $q$ or $\bar{q}$ is true. While

\footnotetext{
${ }^{1}$ An informative voter reveals her private information in her vote whenever the private information is non-conflicting with the true state. Two kinds of informative behaviour is analysed in this work.
} 
doing so, voters know that the combination $\{\bar{p}, \bar{q}\}$ is not possible. The three possible judgment sets are $\{p, q\},\{p, \bar{q}\},\{\bar{p}, q\}$, abbreviated by $p q, p \bar{q}$ and $\bar{p} q$, respectively ${ }^{2}$. Each voter votes for a judgment set in $\mathcal{J}=\{p q, p \bar{q}, \bar{p} q\}$. A collective decision is taken using a voting rule. A voting rule is defined as a function $f: \mathcal{J}^{n} \rightarrow \mathcal{J}$, which maps each voting profile $\mathbf{v}=\left(v_{1}, \ldots, v_{n}\right)$ to a decision $d \equiv f(\mathbf{v})$. Some salient properties of voting rules are defined below:

- Anonymity: For all voting profiles $\left(v_{1}, \ldots, v_{n}\right) \in \mathcal{J}^{n}$ and all permutations $\left(i_{1}, \ldots\right.$, $\left.i_{n}\right)$ of the voters, $f\left(v_{i_{1}}, \ldots, v_{i_{n}}\right)=f\left(v_{1}, \ldots, v_{n}\right)$. Informally, the voters are treated equally.

- Monotonicity: For all voting profiles $\mathbf{v}, \mathbf{v}^{\prime} \in \mathcal{J}^{n}$, if for each $r$ in $f(\mathbf{v})$ the voters who accept $r$ in $\mathbf{v}$ also accept $r$ in $\mathbf{v}^{\prime}$, then $f\left(\mathbf{v}^{\prime}\right)=f(\mathbf{v})$. Informally, additional support for the collectively accepted propositions never reverses the collective acceptance of these propositions.

- Independence: The decision on each proposition $r \in\{p, q\}$ only depends on the votes on $r$. Informally, the group in effect takes two separate votes, one between $p$ and $\bar{p}$ and one between $q$ and $\bar{q}$.

To define the next property, we introduce some notation. Given a voting profile $\mathbf{v}=\left(v_{1}, \ldots, v_{n}\right)$, for each $r \in\{p, q\}$ let $\mathbf{v}_{r}:=\left(v_{1 r}, \ldots, v_{n r}\right)$ be the vector with entities defined as follows: for $i=1, \ldots, n, v_{i r}=1$ if $v_{i}$ contains $r$ and $v_{i r}=0$ if $v_{i}$ contains $\bar{r}$.

- Neutrality: For every voting profile $\mathbf{v}$ and every voting profile $\mathbf{v}^{\prime}$ for which there is no permutation $\left(i_{1}, \ldots, i_{n}\right)$ of the voters with $\left(v_{i_{1}}, \ldots, v_{i_{n}}\right)=\left(v_{1}^{\prime}, \ldots, v_{n}^{\prime}\right)$, if $\mathbf{v}_{r}=\mathbf{v}^{\prime}{ }^{\prime}$ for each $r, r^{\prime} \in\{p, q\}$ with $r \neq r^{\prime}$, then $f$ accepts $r$ in $\mathbf{v}$ if and only if $f$ accepts $r^{\prime}$ in $\mathbf{v}^{\prime}$. Informally, if two voting profiles have the exact same acceptance regime between different propositions, so do the decisions.

\subsubsection{A common preference for true collective judgments}

There is one 'correct' judgment set in $\mathcal{J}$, which we call the state (of the world), denoted by $s$. The state is unobservable by voters. Voters have identical preferences, captured by a common utility function $u: \mathcal{J} \times \mathcal{J} \rightarrow \mathbb{R}$ which maps any decision-state pair $(d, s)$ to its utility $u(d, s)$. The notion of truth-tracking requires the utility to be high if the decision is correct, but details matter. We focus on two natural kinds of preferences:

Simple preferences. The utility function is given by

$$
u(d, s)= \begin{cases}1 & \text { if } d=s \text { (correct decision) } \\ 0 & \text { if } d \neq s \text { (incorrect decision) }\end{cases}
$$

Simple preferences are the simplest candidate for truth-tracking preferences. ${ }^{3}$

\footnotetext{
${ }^{2}$ Similarly, $\{\bar{p}, \bar{q}\}$ is abbreviated by $\bar{p} \bar{q}$.

${ }^{3} \mathrm{~A}$ voter tracks the truth on a proposition $p$ if the following is true: if $p$ were true, the voter would accept $p$ and if $p$ were false, the agent would accept $\bar{p}$ (Nozick, 1981).
} 
Consequentialist preferences. Here, we assume that the decision leads to one of two possible consequences which represents group actions. ${ }^{4}$ This is captured by a consequence function Co which maps the set of possible decisions $\mathcal{J}$ to a two-element set of possible consequences. Consider a market with only one firm, Firm A. Firms $\mathrm{B}$ and $\mathrm{C}$ are interested in entering the market and Firm $\mathrm{B}$ has higher capacity than Firm C. The executive board of firm $\mathrm{C}$ is to make judgments on whether or not firm $\mathrm{A}$ will expand its capacity $(p)$ and firm B will enter the market $(q)$. While doing so, the board members know that if Firm A does not increase capacity, then Firm B will enter the market; hence, $\bar{p} \rightarrow q$. If both issues are judged to be true, then the consequence is 'no market entry' $(\mathrm{Co}(p q)=$ 'no market entry'), while if only one of the issues is judged to be true, the consequence is 'market entry' $\left(\mathrm{Co}(p \bar{q})=\operatorname{Co}(\bar{p} q)=\right.$ 'market entry'). ${ }^{5}$ It turns out that this consequence function with the property $\operatorname{Co}(p q) \neq \operatorname{Co}(p \bar{q})=\operatorname{Co}(\bar{p} q)$ is the only interesting consequence function up to isomorphism. (See Section 2.4 for further discussion.) The consequentialist utility function is given by

$$
u(d, s)= \begin{cases}1 & \text { if } \mathrm{Co}(d)=\mathrm{Co}(s) \text { (correct consequence) } \\ 0 & \text { if } \operatorname{Co}(d) \neq \operatorname{Co}(s) \text { (incorrect consequence) }\end{cases}
$$

\subsubsection{Private information and strategies}

Each voter has a type, which represents private information or evidence about whether $p$ is true and information about whether $q$ is true. A voter's type takes the form of an element of $\mathcal{T}:=\{p q, p \bar{q}, \bar{p} q, \bar{p} \bar{q}\}$, generically denoted by $t$. For instance, the type $t=p \bar{q}$ represents evidence for $p$ and for $\bar{q}$, and the type $t=\bar{p} \bar{q}$ represents evidence for $\bar{p}$ and for $\bar{q}$, which is conflicting information since $\bar{p} \bar{q} \notin \mathcal{J}$. We write $\mathbf{t}=\left(t_{1}, \ldots, t_{n}\right) \in \mathcal{T}^{n}$ for a profile of voters' types.

Nature draws a state-types combination $(s, \mathbf{t})$ in $\mathcal{J} \times \mathcal{T}^{n}$ according to a probability measure denoted Pr. When a proposition $r$ in $\{p, \bar{p}, q, \bar{q}\}$ represents (part of) voter $i$ 's type rather than (part of) the true state, we often write $r_{i}$ for $r$. For instance, $\operatorname{Pr}\left(p_{i} \mid p\right)$ is the probability that voter $i$ has evidence for $p$ given that $p$ is true. By convention, the prior probability of state $s \in \mathcal{J}$ is denoted

$$
\pi_{s}=\operatorname{Pr}(s)
$$

and is assumed to be in the interval $(0,1)$. The probability of getting evidence for $r$ given that $r$ is true is denoted

$$
a_{r}=\operatorname{Pr}\left(r_{i} \mid r\right)
$$

and by assumption belongs to $(1 / 2,1)$ and does not depend on the voter $i$.

By assumption, voters' types are independent given the state. Moreover, given the truth about $p$ (i.e., either $p$ or $\bar{p}$ ), a voter's evidence about $p$ (i.e., either $p_{i}$ or $\bar{p}_{i}$ ) is independent of the truth and the evidence about $q$; and similarly, given the truth about $q$, a voter's evidence about $q$ is independent of the truth and the evidence

\footnotetext{
${ }^{4}$ This two-consequence situation corresponds to problems where the group action is represented by a third proposition - conclusion proposition - which might be true or false. Judging it to be true leads to one of the actions/consequences while judging it to be false leads to the other.

${ }^{5}$ There is still demand left for Firm $\mathrm{C}$ if only one of the companies is in the market.
} 
about $p$. These independence assumptions allow one to express the joint distribution of the state and the types:

$$
\operatorname{Pr}(s, \mathbf{t})=\operatorname{Pr}(s) \times \prod_{i=1}^{n} \operatorname{Pr}\left(t_{i} \mid s\right) .
$$

Here, $\operatorname{Pr}(s)=\pi_{s}$, and the term $\operatorname{Pr}\left(t_{i} \mid s\right)$ is also expressible in terms of our parameters; for instance,

$$
\begin{aligned}
& \operatorname{Pr}\left(p_{i} q_{i} \mid p q\right)=\operatorname{Pr}\left(p_{i} \mid p q\right) \operatorname{Pr}\left(q_{i} \mid p q, p_{i}\right)=\operatorname{Pr}\left(p_{i} \mid p\right) \operatorname{Pr}\left(q_{i} \mid q\right)=a_{p} a_{q} . \\
& \operatorname{Pr}\left(p_{i} \bar{q}_{i} \mid p q\right)=\operatorname{Pr}\left(p_{i} \mid p q\right) \operatorname{Pr}\left(\bar{q}_{i} \mid p q, p_{i}\right)=\operatorname{Pr}\left(p_{i} \mid p\right) \operatorname{Pr}\left(\bar{q}_{i} \mid q\right)=a_{p}\left(1-a_{q}\right)
\end{aligned}
$$

Each voter submits a vote in $\mathcal{J}$ based on his type. A (voting) strategy is a function $\sigma: \mathcal{T} \rightarrow \mathcal{J}$, mapping each type $t \in \mathcal{T}$ to the type's vote $v=\sigma(t)$. We write $\boldsymbol{\sigma}=\left(\sigma_{1}, \ldots, \sigma_{n}\right)$ for a profile of voters' strategies. Together with a voting rule $f$ and a common utility function $u$, we now have a well-defined Bayesian game.

For a given type profile $\mathbf{t} \in \mathcal{T}^{n}$, we call a decision $d \in \mathcal{J}$ efficient if it has maximal expected utility conditional on the full information $\mathbf{t}$. We adapt some common notions of voting behaviour to this framework.

- A strategy $\sigma$ of a voter is mostly informative if $\sigma(t)=t$ for all $t \in \mathcal{T} \backslash\{\bar{p} \bar{q}\}$.

- A strategy $\sigma$ of a voter is informative if $\sigma(t)=t$ for all $t \in \mathcal{T} \backslash\{\bar{p} \bar{q}\}$ and $\sigma(\bar{p} \bar{q}) \in\{p \bar{q}, \bar{p} q\}$.

- A strategy profile $\boldsymbol{\sigma}=\left(\sigma_{1}, \ldots, \sigma_{n}\right)$ is rational if each strategy is a best response to the other strategies, i.e., if the profile is a Nash equilibrium of the corresponding Bayesian game. Hence, each voter maximises the expected utility of the collective decision given the strategies of the other voters.

- A strategy profile $\boldsymbol{\sigma}=\left(\sigma_{1}, \ldots, \sigma_{n}\right)$ is efficient if for every type profile $\mathbf{t}=$ $\left(t_{1}, \ldots, t_{n}\right)$ the resulting decision $d=f\left(\sigma_{1}\left(t_{1}\right), \ldots, \sigma_{n}\left(t_{n}\right)\right)$ is efficient (i.e., has maximal expected utility conditional on full information $\mathbf{t}$ ). Hence, all the information spread across the group is used efficiently: the collective decision is no worse than a decision of a (virtual) social planner who has full information.

A voter with mostly informative strategy votes for her type if her type is nonconflicting, i.e., not $\bar{p} \bar{q}$; while she completely ignores the conflicting evidence $(t=\bar{p} \bar{q})$. In the case of informative strategy, conflicting evidence is followed partly. Unless we particularly mean one of these strategies, we say informative behaviour to refer to them. Note that rationality and efficiency refer to a whole profile of strategies.

We make two assumptions to avoid trivialities. First, we exclude the degenerate case where some decision in $\mathcal{J}$ is not efficient for any type profile. Hence, each decision is efficient for at least one type profile. Second, we exclude efficiency ties, i.e., those special parameter combinations such that some type profile leads to different efficient decisions (with different consequences when we assume consequentialist preferences). Hence, we exclude those instances where a voter is indifferent between two decisions except in the case that these decisions lead to the same consequence. 


\section{$2.2 \quad$ A general (im)possibility}

How should the voting rule be designed so that it leads to efficient decisions as well as simple-minded, truthful voting behaviour in equilibrium? The objective of the chapter is to answer this question. By simple-minded, truthful behaviour, we mean informative behaviour. A voting rule encourages simple-minded behaviour if it makes informative voting rational. ${ }^{6}$ If informative voting is both rational and efficient, the objective is reached. Note that neither an informative strategy nor a mostly informative strategy is unique. Informative voting being efficient means that for any given type profile $\mathbf{t}$, every profile of corresponding informative strategies is efficient. By the following theorem, our objective is reduced to finding out when informative voting is efficient.

Theorem 1 For any common utility function $u: \mathcal{J}^{2} \rightarrow \mathbb{R}$, and for any voting rule $f: \mathcal{J}^{n} \rightarrow \mathcal{J}$, if a strategy profile is efficient, then it is rational.

This result applies to any kind of common preferences. Is it always possible to find a voting rule which makes informative voting efficient, hence, rational? The next theorem answers this question.

Theorem 2 Consider an arbitrary common utility function $u: \mathcal{J}^{2} \rightarrow \mathbb{R}$. There exists no voting rule for which mostly informative voting is efficient.

This theorem states that there is no voting rule which achieves efficient information aggregation for every possible mostly informative strategy profile. This result comes as a surprise when one considers the single-issue setting and multi-issue setting with no interconnections, where there is always a voting rule for which informative voting is efficient. This contrast comes from the fact that the notion of informative voting is not very clear in the current setting, since there is no straightforward way of adapting informativeness. It is clear what a simple-minded voter should do when she receives non-conflicting evidence about the state of the world, but what about the conflicting evidence, $\bar{p} \bar{q}$ ? Here, voters with the mostly informative strategy have no restriction upon receiving type $\bar{p} \bar{q}$. This leads to the question of whether the impossibility persists when one considers informativeness differently. Let us now consider informative voting. Voters holding informative strategy follow the conflicting evidence partly. Does this additional requirement lead to any possibility for efficient information aggregation? The result is yes, and it is formalized by the coming theorem. To state the theorem, we first introduce some notation and a condition.

Given a type profile $\mathbf{t}=\left(t_{1}, \ldots, t_{n}\right)$, let $\mathbf{t}_{p q}=\left(t_{p q_{1}}, \ldots, t_{p q_{n}}\right)$ be the vector with entities defined for $i=1, \ldots, n$ as $t_{p q_{i}}=1$ if $t_{i}=p q$ and $t_{p q_{i}}=0$ otherwise. ${ }^{7}$ Given a voting profile $\mathbf{v}, \mathbf{v}_{p q}$ is defined similarly. At this point, it is useful to remark that for any type profile, there is an efficient decision.

\footnotetext{
${ }^{6}$ Here, by informative voting, we mean informative behaviour - with mostly informative strategy or informative strategy - in general. This chapter analyses the case with informative strategy and the case with mostly informative strategy separately.

${ }^{7}$ For instance, for the type profiles $\mathbf{t}=(p \bar{q}, p q, \bar{p} \bar{q})$ and $\mathbf{t}^{\prime}=(\bar{p} q, p q, \bar{p} q), \mathbf{t}_{p q}=\mathbf{t}_{p q}^{\prime}=(0,1,0)$.
} 
Condition 1: For any $\mathbf{t}, \mathbf{t}^{\prime} \in \mathcal{T}^{n}$ with $\mathbf{t}_{p q}=\mathbf{t}_{p q}^{\prime}$, there is a decision $d \in \mathcal{J}$ which is efficient for both $\mathbf{t}, \mathbf{t}^{\prime}$.

Theorem 3 Consider an arbitrary common utility function $u: \mathcal{J}^{2} \rightarrow \mathbb{R}$. There exists a voting rule for which informative voting is efficient if and only if Condition 1 holds.

When we require an informative voter to follow the conflicting evidence partly instead of completely ignoring it, efficient information aggregation is possible when some condition on the model parameters is satisfied. How does the voting rule making efficient information aggregation possible look like? The answer depends on how exactly the utility function is specified. We can say more about this rule only when we focus on specific kind of preferences.

Having a general impossibility for informative voting with mostly informative strategy, we focus on informative voting for the rest of the chapter. To see how strong condition 1 is, one has to narrow focus on specific preferences. We study the two natural kinds of preferences - simple and consequentialist preferences - in the following subsections.

\subsection{Simple preferences}

We start by addressing simple preferences. Under simple preferences, correct decisions are preferred to incorrect ones without further sophistication. By narrowing down the focus on simple preferences, can we say more about the voting rule which makes informative voting efficient under Condition 1 and obtain a more specific result than the existential claim in Theorem 3? For simple preferences, we obtain the following impossibility.

Theorem 4 Under simple preferences, there exists no voting rule for which informative voting is efficient.

It turns out that Condition 1 is never satisfied under simple preferences. In addition to the impossibility of efficient information aggregation with informative strategy stated in Theorem 2, efficient information aggregation with informative strategy is impossible under simple preferences. Does the impossibility persist under consequentialist preferences? The next subsection addresses this question.

\subsection{Consequentialist preferences}

We now turn to consequentialist preferences. We consider situations where the decision leads to one of two possible consequences. Such problems are very common in practice and widely studied in the judgment aggregation literature, where the two possible consequences are represented by conclusion propositions, $c$ and $\bar{c}$. The decision leads to either acceptance of the conclusion proposition or rejection of it ${ }^{8}$.

\footnotetext{
${ }^{8}$ Consider the lead example of judgment aggregation: a jury is to decide whether the defendant has broken the contract $(p)$ or not $(\bar{p})$ and whether the contract is legally valid $(q)$ or not $(\bar{q})$. The defendant is convicted if and only if both propositions are collectively accepted. The consequence function here is encoded by $c \leftrightarrow(p \wedge q)$.
} 
Consequence functions which lead all decisions to the same consequence are degenerate and uninteresting. If the consequence function depends only on the decision between $p$ and $\bar{p}$, or only on the decision between $q$ and $\bar{q}$, then the decision problem reduces to a problem with a single proposition-negation pair which has already been studied in the literature. Therefore, there is only one interesting consequence function up to isomorphism, and this function has the property $\operatorname{Co}(p q) \neq \operatorname{Co}(p \bar{q})=\operatorname{Co}(\bar{p} q)$.

To state our result, we first define two coefficients:

$$
\begin{aligned}
& A:=\pi_{p \bar{q}}\left(\frac{1-a_{\bar{q}}}{a_{q}}\right)^{n}+\pi_{\bar{p} q}\left(\frac{1-a_{\bar{p}}}{a_{p}}\right)^{n-1} \frac{a_{\bar{p}}}{1-a_{p}} \\
& B:=\pi_{p \bar{q}}\left(\frac{1-a_{\bar{q}}}{a_{q}}\right)^{n-1} \frac{a_{\bar{q}}}{1-a_{q}}+\pi_{\bar{p} q}\left(\frac{1-a_{\bar{p}}}{a_{p}}\right)^{n}
\end{aligned}
$$

Theorem 5 Under consequentialist preferences, the following statements are equivalent:

(a) There exists a voting rule for which informative voting is efficient.

(b) $A, B>\pi_{p q}$.

(c) $p q$ is the efficient decision only for the type profile $\mathbf{t}=(p q, \ldots, p q)$.

Unlike under simple preferences, efficient information aggregation is possible under consequentialist preferences, if $p q$ is the efficient decision only when there is overwhelming evidence for $p q$. This is satisfied when the prior probability of $p q$ is sufficiently low compared to prior probabilities of $p \bar{q}$ and $\bar{p} q$. For instance, if $\pi_{p q}=\pi_{p \bar{q}}=\pi_{\bar{p} q}=0.7, a_{p}=a_{q}=a_{\bar{p}}=a_{\bar{q}}=0.6$ and $n=3$, no voting rule makes informative voting efficient, whereas if instead $\pi_{p q}=0.6$, such a voting rule exists. Now comes the main question: how do such rules look like? Let us call the condition stated at Theorem 5(b) Condition 2. We start by a simple characterization of voting rules which make informative voting efficient.

Proposition 1 Assume consequentialist preferences and Condition 2. A voting rule $f$ makes informative voting efficient if and only if for every voting profile $\mathbf{v} \in \mathcal{J}^{n}$, the decision $f(\mathbf{v})$ is $p q$ if $\mathbf{v}=(p q, \ldots, p q)$ and in $\{p \bar{q}, \bar{p} q\}$ otherwise.

While some of these voting rules making informative voting efficient are anonymous, monotonic and neutral, some of them fail to satisfy any of these properties. The number of votes for a proposition $r$ in a voting profile $\mathbf{v}$ is written $n_{r}^{\mathbf{v}}$. For $n=5$, two examples of anonymous and monotonic rules are given in the figure below: 

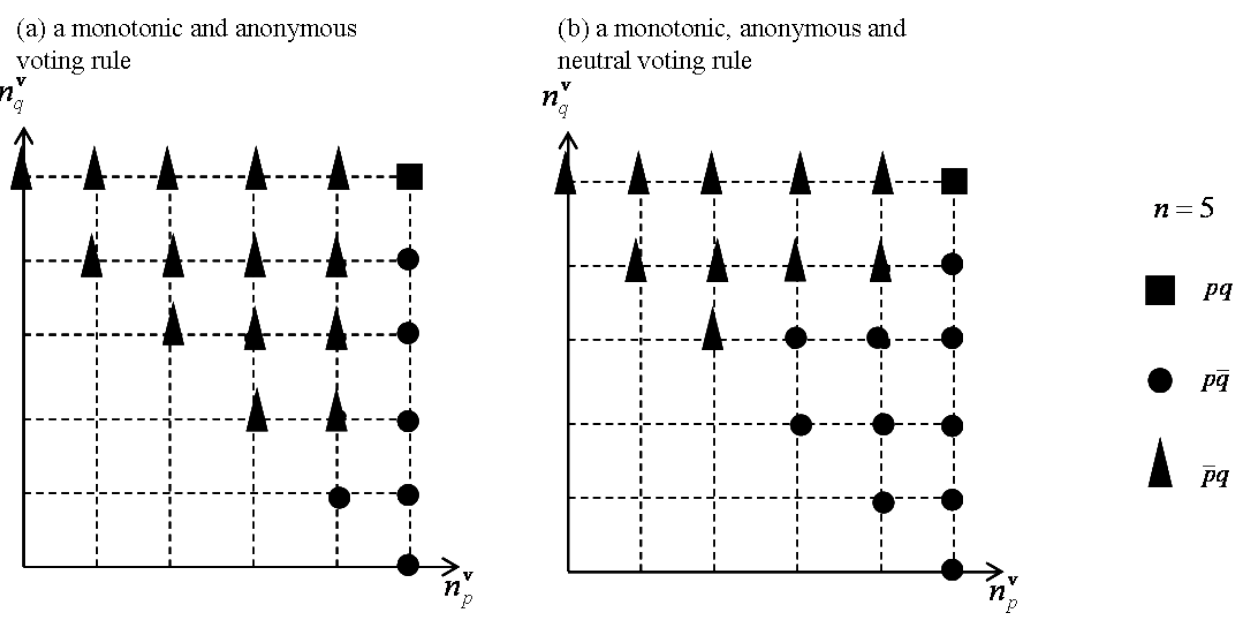

Figure 1: Two examples of voting rules given by Proposition 1 for $n=5$

Figure 1(b) shows a neutral voting rule in addition to being anonymous and monotonic. This voting rule belongs to a class of voting rules defined by the following conditions. For each $\mathbf{v} \in \mathcal{J}^{n}$,

$$
\begin{aligned}
& f(\mathbf{v})=p q \Longleftrightarrow n_{p}^{\mathbf{v}}=n_{q}^{\mathbf{v}}=n \\
& f(\mathbf{v})=p \bar{q} \text { if } n_{p}^{\mathbf{v}}>n_{q}^{\mathbf{v}} \\
& f(\mathbf{v})=\bar{p} q \text { if } n_{p}^{\mathbf{v}}<n_{q}^{\mathbf{v}} \\
& f(\mathbf{v}) \in\{p \bar{q}, \bar{p} q\} \text { if } n_{p}^{\mathbf{v}}=n_{q}^{\mathbf{v}}<n
\end{aligned}
$$

By this class of voting rules defined by (3-6), we characterize the class of anonymous, monotonic and neutral voting rules making informative voting efficient under Condition 2.

Theorem 6 Assume consequentialist preferences and Condition 2. A voting rule $f$ makes informative voting efficient and is anonymous, monotonic and neutral if and only if $f$ is defined by (3)-(6).

Besides satisfying nice properties, these rules are reasonably practical and natural. We have now shown the necessary and sufficient conditions for the existence of a mechanism for efficient information aggregation, and characterized the mechanism with natural properties. Among the aggregation possibilities, anonymity, monotonicity and neutrality can be attained if required. What about independence? The next section answers this question.

\section{Consistency and quota rules under consequentialist preferences}

Quota rules are very natural and common among various voting rules. Under quota rules, separate votes are taken on each proposition using acceptance thresholds. In 
the previous section, we have seen that under consequentialist preferences, efficient information aggregation is possible in an anonymous, monotonic and neutral way when some condition on the model parameters is satisfied. Quota rules are monotonic, anonymous and independent, but not necessarily neutral. ${ }^{9}$ This section examines the possibility of efficient information aggregation with quota rules under consequentialist preferences. To do so, one has to re-define a voting rule and utility function. The model described in the previous section applies to this section with exceptions described below.

Let $\mathcal{J}^{*}:=\{p q, p \bar{q}, \bar{p} q, \bar{p} \bar{q}\}$. For this section, a voting rule is a function $f: \mathcal{J}^{n} \rightarrow$ $\mathcal{J}^{*}$, mapping each voting profile $\mathbf{v}=\left(v_{1}, \ldots, v_{n}\right)$ to a decision $d \equiv f(\mathbf{v})$. A voting rule $f$ is called 'consistent' if it never returns $\bar{p} \bar{q}$.

The consequentialist utility function is now given as $u: \mathcal{J}^{*} \times \mathcal{J} \rightarrow \mathbb{R}$, mapping any decision-state pair $(d, s) \in \mathcal{J}^{*} \times \mathcal{J}$ to its utility $u(d, s)$. By assumption, the decision $\bar{p} \bar{q}$ never has the correct consequence, hence, $\operatorname{Co}(\bar{p} \bar{q}) \neq \operatorname{Co}(s)$. The consequentialist utility function is given by $(2)$.

A quota rule is given by two thresholds $m_{p}, m_{q} \in\{0,1, \ldots, n\}$, and for each voting profile it accepts $p[q]$ if and only if at least $m_{p}\left[m_{q}\right]$ voters accept it in the profile. Quota rules are characterized by anonymity, monotonicity and independence. The remark below follows from Theorem 2(c) in Dietrich and List (2007) and gives the necessary and sufficient conditions for a quota rule to be consistent under the given agenda.

Remark 1 A quota rule is consistent if and only if $m_{p}+m_{q} \leq n+1$.

The proof of this remark follows from the fact that there can be at most $n$ votes in total for $\bar{p}$ and $\bar{q}$ (since $\bar{p} \bar{q} \notin \mathcal{J}$ ). It is easy to see that consistency is attained whenever $m_{p}+m_{q} \leq n$. Moreover, if $m_{p}+m_{q}=n+1$, the number of $p$ votes or the number of $q$ votes exceeds the acceptance threshold, so, the resulting decision is never $\bar{p} \bar{q}$. Note that if a voting rule leads to efficient decisions, then it is consistent. So, as long as efficiency is guaranteed, consistency follows. Among all the rules making informative voting efficient, is there a quota rule? The theorem below answers this question.

Theorem 7 Under consequentialist preferences, there exists no quota rule making informative voting efficient.

A consistent quota rule is always available by Remark 1 regardless of the model parameters or the utility function chosen. However, there is no possibility for efficient information aggregation using quota rules.

\section{Conclusion}

We consider a model where a group of voters with common interests wants to form collective judgments over two propositions which are mutually interconnected. Each of these propositions is factually true or false, but the truth value is unknown to voters. Each voter has a type representing evidence about what the true state might

\footnotetext{
${ }^{9}$ Whenever the acceptance thresholds for propositions are equal, they turn out to be neutral.
} 
be and this is private information. We study the problem of efficient information aggregation when propositions are mutually interconnected. The results depend particularly on how the utility function is specified. It turns out that a voting rule which makes informative voting efficient does not exist under simple preferences while such a rule exists under consequentialist preferences if some condition relating the model parameters and the utility function is satisfied. We want to design a voting rule which make every possible informative strategy profile efficient. Under simple preferences, it is of course possible to find a voting rule which makes some informative strategy profile efficient. However, we believe a voting rule which sometimes makes informative voting efficient is not really interesting.

We leave unanswered whether these results persist when conflicting private information $-\bar{p} \bar{q}$ in this case - is not allowed. In that case, we can no longer assume that a voter's evidence about one proposition is independent of the evidence about the other proposition conditional on the truth. For instance, if a voter has $\bar{p}$ in her type, she must have $q$. Informative strategy is then defined as direct revelation of types, and there is a unique informative strategy profile given a type profile.

\section{$5 \quad$ References}

Ahn, D., Oliveros S. (2011) Condorcet Jur(ies) Theorem. Mimeo, Berkeley University

Ahn, D., Oliveros S. (2012) Combinatorial voting. Econometrica, 80(1): 89141

Austen-Smith, D., Banks, J. (1996) Information aggregation, rationality, and the Condorcet jury theorem. The American Political Science Review 90: 34-45

Austen-Smith, D., Feddersen, T. (2005) Deliberation and Voting Rules, in AustenSmith, D. and J. Duggan (eds) Social Choice and Strategic Decisions: Essays in Honor of Jeffrey S. Banks, Berlin: Springer

Austen-Smith, D., Feddersen, T. (2006) Deliberation, preference uncertainty and voting rules. American Political Science Review 100(2): 209-217

Bovens, L., Rabinowicz, W. (2006) Democratic answers to complex questions: an epistemic perspective. Synthese 150(1): 131-153

Bozbay, I., Dietrich, F., Peters, H. (2011) Judgment aggregation in search for the truth. Mimeo, Maastricht University

Coughlan, P. (2000) In defense of unanimous jury verdicts: mistrials, communication and strategic voting. The American Political Science Review 94(2): 375-393

De Clippel G., Eliaz K. (2011) Premise versus outcome-based information aggregation. Mimeo, Brown University

Dietrich, F. (2006) Judgment aggregation: (im)possibility theorems. Journal of Economic Theory 126(1): 286-298 
Dietrich, F. (2007) A generalised model of judgment aggregation. Social Choice and Welfare 28(4): 529-565

Dietrich, F. (2010) The possibility of judgment aggregation on agendas with subjunctive implications. Journal of Economic Theory 145(2): 603-638

Dietrich, F., List, C. (2007a) Arrow's theorem in judgment aggregation. Social Choice and Welfare 29(1): 19-33

Dietrich, F., List, C. (2007b) Strategy-proof judgment aggregation. Economics and Philosophy 23: 269-300

Dietrich, F., List, C. (2007c) Judgment aggregation by quota rules: majority voting generalized. Journal of Theoretical Politics 19(4): 391-424

Dietrich, F., List, C. (2008) Judgment aggregation without full rationality. Social Choice and Welfare 31: 15-39

Dietrich, F., Mongin, P. (2010) The premise-based approach to judgment aggregation. Journal of Economic Theory 145(2): 562-582

Dokow, E., Holzman, R. (2010a) Aggregation of binary evaluations. Journal of Economic Theory 145(2): 495-511

Dokow, E., Holzman, R. (2010b) Aggregation of binary evaluations with abstentions. Journal of Economic Theory 145(2): 544-561

Duggan, J., Martinelli, C. (2001) A Bayesian model of voting in juries. Games and Economic Behavior 37(2): 259-294

Feddersen, T., Pesendorfer, W.(1997) Information aggregation and voting behaviour in elections. Econometrica 65(5): 1029-1058

Feddersen, T., Pesendorfer, W. (1998) Convicting the innocent: the inferiority of unanimous jury verdicts under strategic voting. The American Political Science Review 92(1): 23-15

Gerardi, D., (2000) Jury verdicts and preference diversity. American Political Science Review 94: 395-406

Guilbaud, G. (1952) Les théories de l'intérêt général et le problème logique de l'agrégation. Économie Appliquée 5: 501-584

List, C. (2005) The probability of inconsistencies in complex collective decisions. Social Choice and Welfare 24(1): 3-32

List, C., Pettit, P. (2002) Aggregating sets of judgments: an impossibility result. Economics and Philosophy 18(1): 89-110

List, C., Pettit, P. (2011) Group Agency: The Possibility, Design and Status of Corporate Agents. Oxford University Press 
List, C., Polak, B. (2010) Introduction to judgment aggregation. Journal of Economic Theory 145(2): 441-466

Nehring, K., Puppe, C. (2002) Strategy-proof social choice on single-peaked domains: possibility, impossibility and the space between. Working paper, University of California at Davis

Nehring, K., Puppe, C. (2007) The structure of strategy-proof social choice. Part I: General characterization and possibility results on median spaces. Journal of Economic Theory 135: 269-305

Nehring, K. Puppe, C. (2008) Consistent judgement aggregation: the truth-functional case. Social Choice and Welfare 31: 41-57

Nehring, K., Puppe, C. (2010) Abstract Arrovian aggregation. Journal of Economic Theory 145(2): 467-494

Nozick, R. (1981) Philosophical explanations. Oxford University Press

Wilson R (1975) On the theory of aggregation. Journal of Economic Theory 10: 89-99

\section{A Appendix: proofs}

We begin by some preliminary derivations and then prove the results

\section{A.1 Preliminary derivations}

The joint probability of a state-types vector $(s, \mathbf{t})=\left(s_{p} s_{q}, t_{1 p} t_{1 q}, \ldots, t_{n p} t_{n q}\right) \in \mathcal{J}^{n+1}$ is

$$
\operatorname{Pr}(s, \mathbf{t})=\operatorname{Pr}(s) \operatorname{Pr}(\mathbf{t} \mid s)=\operatorname{Pr}(s) \prod_{i} \operatorname{Pr}\left(t_{i} \mid s\right)=\operatorname{Pr}\left(s_{p}\right) \operatorname{Pr}\left(s_{q}\right) \prod_{i} \operatorname{Pr}\left(t_{i p} \mid s_{p}\right) \operatorname{Pr}\left(t_{i q} \mid s_{q}\right),
$$

where the last two equations follow from independence assumptions.

The probability of the three states in $\mathcal{J}$ conditional on the full information $\mathbf{t} \in \mathcal{J}^{n}$ is given as follows, where $k:=n_{p}^{\mathbf{t}}$ and $l:=n_{q}^{\mathbf{t}}$ :

$$
\begin{aligned}
\operatorname{Pr}(p q \mid \mathbf{t}) & =\frac{\pi_{p q} a_{p}^{k}\left(1-a_{p}\right)^{n-k} a_{q}^{l}\left(1-a_{q}\right)^{n-l}}{\operatorname{Pr}(\mathbf{t})} \\
\operatorname{Pr}(p \bar{q} \mid \mathbf{t}) & =\frac{\pi_{p \bar{q}} a_{p}^{k}\left(1-a_{p}\right)^{n-k}\left(1-a_{\bar{q}}\right)^{l} a_{\bar{q}}^{n-l}}{\operatorname{Pr}(\mathbf{t})} \\
\operatorname{Pr}(\bar{p} q \mid \mathbf{t}) & =\frac{\pi_{\bar{p} q}\left(1-a_{\bar{p}}\right)^{k} a_{\bar{p}}^{n-k} a_{q}^{l}\left(1-a_{q}\right)^{n-l}}{\operatorname{Pr}(\mathbf{t})} .
\end{aligned}
$$




\section{A.2 Proofs.}

Proof of Theorem 1. Consider any voting rule $f: \mathcal{J}^{n} \rightarrow \mathcal{J}$ and any efficient strategy profile $\boldsymbol{\sigma}$. Consider any voter $i$ and type $t_{i} \in \mathcal{T}$. To show that $\boldsymbol{\sigma}$ is rational, one has to show that $i$ 's vote $\sigma_{i}\left(t_{i}\right)$ maximizes her expected utility conditional on $t_{i}$. This follows from the fact that voters share common preferences. Since the resulting decision is efficient, it maximizes the expected utility of each voter. Thus, $\boldsymbol{\sigma}$ is rational.

Proof of Theorem 2. Consider a voting rule $f: \mathcal{J}^{n} \rightarrow \mathcal{J}$. Suppose for a contradiction $f$ makes informative voting efficient. Consider the type profile $\mathbf{t}=(\bar{p} \bar{q}, \ldots, \bar{p} \bar{q})$, where all voters have the type $\bar{p} \bar{q}$. Then, the set of all voting profiles which may result from informative voting is $\mathcal{J}^{n}$. Since informative voting is efficient, for each $\mathbf{v} \in \mathcal{J}^{n}$, $f(\mathbf{v})$ is efficient given $\mathbf{t}$. Then, it follows that for all type profiles in $\mathcal{T}^{n}, f(\mathbf{v})$ is efficient. This either means that some decision in $\mathcal{J}$ is not efficient for any type profile which contradicts to non-degeneracy assumption, or all decisions in $\mathcal{J}$ are always efficient for any type profile which contradicts to no efficiency ties assumption. ${ }^{10}$

Proof of Theorem 3. To start with, we introduce some notation. Given a voting profile $\mathbf{v}$, let $\Theta(\mathbf{v})$ denote the set of all type profiles which possibly lead to $\mathbf{v}$ under informative voting. Given a type profile $\mathbf{t}$, let $\Omega(\mathbf{t})$ denote the set of all voting profiles which possibly result from $\mathbf{t}$ under informative voting. Consider a voting rule $f: \mathcal{J}^{n} \rightarrow \mathcal{J}$.

First, let Condition 1 hold. Suppose there is an exogenously given ordering of judgment sets, and let $f$ be the following voting rule: for all $\mathbf{v} \in \mathcal{J}^{n}, f(\mathbf{v})=d \Longleftrightarrow$ $d$ is the highest ordered decision among all decisions which are efficient for some $\mathbf{t} \in \Theta(\mathbf{v})$. Consider any type profile $\hat{\mathbf{t}} \in \mathcal{T}^{n}$ and suppose informative voting. We want to show that $\left(^{*}\right)$ for each $\mathbf{v} \in \Omega(\hat{\mathbf{t}}), f(\mathbf{v})$ is efficient for $\hat{\mathbf{t}}$. Let $\mathbf{v} \in \Omega(\hat{\mathbf{t}})$. One can show that all type profiles in $\Theta(\mathbf{v})$ share the same subvector restricted to $p q$. Since Condition 1 holds, there is some decision $d$ which is efficient for all $\mathbf{t} \in \Theta(\mathbf{v})$, including $\hat{\mathbf{t}}$. It follows from Condition 1 that if any other decision $d^{\prime} \neq d$ is efficient for some $\mathbf{t} \in \Theta(\mathbf{v})$, it is efficient for all $\mathbf{t} \in \Theta(\mathbf{v})$. Then, $\left(^{*}\right)$ holds.

Conversely, let $f$ make informative voting efficient. Let $\mathbf{t}, \mathbf{t}^{\prime}$ be two type profiles in $\mathcal{T}^{n}$ with $\mathbf{t}_{p q}=\mathbf{t}_{p q}^{\prime}$. One has to show that $\left(^{*}\right)$ there is $d \in \mathcal{J}$ which is efficient for both $\mathbf{t}, \mathbf{t}^{\prime}$. By construction, for each $\mathbf{v} \in \Omega(\mathbf{t}), \mathbf{t}^{\prime} \in \Theta(\mathbf{v})$; and similarly, for each $\mathbf{v}^{\prime} \in \Omega\left(\mathbf{t}^{\prime}\right), \mathbf{t} \in \Theta\left(\mathbf{v}^{\prime}\right)$. Then, $f(\mathbf{v})$ must be efficient for $\mathbf{t}^{\prime}$ (as well as $\mathbf{t}$ ) and $f\left(\mathbf{v}^{\prime}\right)$ must be efficient for $\mathbf{t}$ (as well as $\mathbf{t}^{\prime}$ ) since informative voting is efficient. So, $(*)$ holds.

Proof of Theorem 4. By Theorem 2, it is sufficient to show that Condition 1 never holds under simple preferences. Suppose for a contradiction, it holds. Consider the two type profiles $\mathbf{t}=(p \bar{q}, \ldots, p \bar{q})$ and $\mathbf{t}^{\prime}=(\bar{p} q, \ldots, \bar{p} q)$. Since $\mathbf{t}_{p q}=\mathbf{t}_{p q}^{\prime}$ and Condition 1 holds, there is a decision which is efficient for both profiles. By non-degeneracy

\footnotetext{
${ }^{10}$ For the case where the consequence function is defined in such a way that all three judgment sets in $\mathcal{J}$ lead to the same consequence, the second assumption is not violated. In such situations, there is no decision making problem since all decisions are equally good for each voter. Such utility functions are excluded.
} 
assumption, $p \bar{q}$ must be efficient for $\mathbf{t}$ since otherwise $p \bar{q}$ wouldn't be efficient for any type profile which contradicts to non-degeneracy assumption. Similarly, $\bar{p} q$ must be efficient for $\mathbf{t}^{\prime}$. Hence, $p \bar{q}$ and $\bar{p} q$ are both efficient given $\mathbf{t}$ or $\mathbf{t}^{\prime}$, which contradicts to no-efficiency ties assumption.

Proof of Theorem 5. Let the statement in (b) be called Condition 2.

(1) We first prove that (c) implies (a) and (b). Assume Condition 2 holds. This implies that Condition 1 holds. By Theorem 3, there is a voting rule which makes informative voting efficient. Let $\mathbf{t}, \mathbf{t}^{\prime}$ be type profiles with one $p \bar{q}$ and one $\bar{p} q$ respectively while each of the rest of the types is $p q$. Without loss of generality, let $\mathbf{t}=(p q, \ldots, \bar{p} q)$ and $\mathbf{t}^{\prime}=(p q, \ldots, p \bar{q})$. By Condition $2, p \bar{q}, \bar{p} q$ are both efficient for each of the type profiles. Using (7) and (9), we can write the following:

$$
\begin{aligned}
& E(u(p \bar{q}, S) \mid \mathbf{t})>E(u(p q, S) \mid \mathbf{t}) \\
\Leftrightarrow & \pi_{p \bar{q}} a_{p}^{n-1}\left(1-a_{p}\right)\left(1-a_{\bar{q}}\right)^{n}+\pi_{\bar{p} q}\left(1-a_{\bar{p}}\right)^{n-1} a_{\bar{p}} a_{q}^{n}>\pi_{p q} a_{p}^{n-1}\left(1-a_{p}\right) a_{q}^{n} \\
\Leftrightarrow & \pi_{p \bar{q}}\left(\frac{1-a_{\bar{q}}}{a_{q}}\right)^{n}+\pi_{\bar{p} q}\left(\frac{1-a_{\bar{p}}}{a_{p}}\right)^{n-1}\left(\frac{a_{\bar{p}}}{1-a_{p}}\right)>\pi_{p q} .
\end{aligned}
$$

Similarly,

$$
\begin{aligned}
& E\left(u(p \bar{q}, S) \mid \mathbf{t}^{\prime}\right)>E\left(u(p q, S) \mid \mathbf{t}^{\prime}\right) \\
\Leftrightarrow & \pi_{p \bar{q}} a_{p}^{n}\left(1-a_{\bar{q}}\right)^{n-1} a_{\bar{q}}+\pi_{\bar{p} q}\left(1-a_{\bar{p}}\right)^{n} a_{q}^{n-1}\left(1-a_{q}\right)>\pi_{p q} a_{p}^{n} a_{q}^{n-1}\left(1-a_{q}\right) \\
\Leftrightarrow & \pi_{p \bar{q}}\left(\frac{1-a_{\bar{q}}}{a_{q}}\right)^{n-1}\left(\frac{a_{\bar{q}}}{1-a_{q}}\right)+\pi_{\bar{p} q}\left(\frac{1-a_{\bar{p}}}{a_{p}}\right)^{n}>\pi_{p q} .
\end{aligned}
$$

So, $A, B>\pi_{p q}$.

(2) We now prove that (a) implies (c). Consider a voting rule $f: \mathcal{J}^{n} \rightarrow \mathcal{J}$ and suppose $f$ makes informative voting efficient. By Theorem 3, Condition 1 holds. Given a type profile $\mathbf{t} \in \mathcal{T}^{n}$, let $\Gamma(\mathbf{t})$ denote the set of type profiles which have the same subvector on $p q$ as in $\mathbf{t}$. Recall that the number of occurrences for a proposition $r$ in a type profile $\mathbf{t}$ is written $n_{r}^{\mathbf{t}}$. Now, take a type profile $\hat{\mathbf{t}} \in \mathcal{T}^{n}$ with $k$ times $p q$ where $1 \leq k<n$. The proof proceeds in several steps.

Claim 1: There is a type profile $\mathbf{t} \in \Gamma(\hat{\mathbf{t}})$ with $n_{p}^{\mathbf{t}}=k$ and $n_{q}^{\mathbf{t}}=k$.

Any type profile with $k$ times $p q$ and $n-k$ times $\bar{p} \bar{q}$ satisfies this condition and one of these type profiles is obviously in $\Gamma(\hat{\mathbf{t}})$. Now, take $\tilde{\mathbf{t}} \in \mathcal{T}^{n}$ with $k-1$ times $p q$.

Claim 2: There is a type profile $\mathbf{t} \in \Gamma(\tilde{\mathbf{t}})$ with $n_{p}^{\mathbf{t}}=k$ and $n_{q}^{\mathbf{t}}=k$.

One can easily see there is always a type profile with the exact same $p q$ structure as $\tilde{\mathbf{t}}$ and with only one occurrence of $p \bar{q}$ and only one occurrence of $\bar{p} q$.

Claim 3: Under consequentialist preferences, for all $\mathbf{t}, \mathbf{t}^{\prime} \in \mathcal{T}^{n}$ with $n_{p}^{\mathbf{t}}=n_{p}^{\mathbf{t}^{\prime}}$ and $n_{q}^{\mathbf{t}}=n_{q}^{\mathbf{t}^{\prime}}, E(u(d, S) \mid \mathbf{t})=E\left(u(d, S) \mid \mathbf{t}^{\prime}\right)$ for each $d \in \mathcal{J}$.

The claim follows from the expressions (7)-(9). By Condition 1, there is a decision $d \in \mathcal{J}$ which is efficient for all $\mathbf{t} \in \Gamma(\hat{\mathbf{t}})$. Similarly, there is a decision $d \in \mathcal{J}$ which is efficient for all $\mathbf{t} \in \Gamma(\tilde{\mathbf{t}})$. Combining Claim 1, 2 and 3, one obtains that the same decision $d \in \mathcal{J}$ is efficient for all $\mathbf{t} \in \Gamma(\hat{\mathbf{t}})$ and all $\mathbf{t} \in \Gamma(\tilde{\mathbf{t}})$. Since this is true for all $k$ with $1 \leq k<n$, there is a decision $d$ which is efficient for all $\mathbf{t} \in \mathcal{T}^{n} \backslash\{(p q, \ldots, p q)\}$. 
By non-degeneracy assumption, $p q$ is efficient for $\mathbf{t}=(p q, \ldots, p q)$. Hence, this decision must be in $\{p \bar{q}, \bar{p} q\}$ since otherwise $p q$ would be efficient for all type profiles which contradicts to non-degeneracy assumption. Since $E(u(p \bar{q}, S) \mid \mathbf{t})=E(u(\bar{p} q, S) \mid \mathbf{t})$ for all $\mathbf{t}$, both $p \bar{q}$ and $\bar{p} q$ are efficient for all $\mathbf{t} \in \mathcal{T}^{n} \backslash\{(p q, \ldots, p q)\}$. Hence, Condition 2 holds.

(3) We finally prove that (b) implies (c). Let $A, B>\pi_{p q}$. To show that Condition 2 holds, we first show the following claim.

Claim 4: The expected utility of $p q$ given a type profile $\mathbf{t}$ is an increasing function of $n_{p}^{\mathbf{t}}$ and $n_{q}^{\mathbf{t}}$.

The claim follows from the definition of the utility function and from $\operatorname{Pr}(S=p q \mid \mathbf{t})$ being an increasing function of $n_{p}^{\mathbf{t}}$ and $n_{q}^{\mathbf{t}}$. Let $\mathbf{t}, \mathbf{t}^{\prime} \in \mathcal{T}^{n}$ be type profiles with one $p \bar{q}$ and one $\bar{p} q$ respectively while each of the rest of the types is $p q$. Without loss of generality, let $\mathbf{t}=(p q, \ldots, \bar{p} q)$ and $\mathbf{t}^{\prime}=(p q, \ldots, p \bar{q})$. By (7) and (9), one has $E(u(p \bar{q}, S) \mid \mathbf{t})>$ $E(u(p q, S) \mid \mathbf{t})$ and $E\left(u(p \bar{q}, S) \mid \mathbf{t}^{\prime}\right)>E\left(u(p q, S) \mid \mathbf{t}^{\prime}\right)$. By the claim, it follows that $E(u(p \bar{q}, S) \mid \mathbf{t})=E(u(\bar{p} q, S) \mid \mathbf{t})>E(u(p q, S) \mid \mathbf{t})$ for all $\mathbf{t} \in \mathcal{T}^{n} \backslash\{(p q, \ldots, p q)\}$ which means $p \bar{q}, \overline{p q}$ are efficient for each $\mathbf{t} \in \mathcal{T}^{n} \backslash\{(p q, \ldots, p q)\}$. Thus, Condition 2 holds.

Proof of Proposition 1. Consider a voting rule $f: \mathcal{J}^{n} \rightarrow \mathcal{J}$. Proof if the 'if' part is obvious and left to the reader. To show converse, let $f$ make informative voting efficient. Since Condition 2 holds, for all voting profiles obtained by informative voting from any $\mathbf{t} \in \mathcal{T}^{n} \backslash\{(p q, \ldots, p q)\}, f(\mathbf{v}) \in\{p \bar{q}, \bar{p} q\}$. By non-degeneracy assumption, $p q$ is efficient for $\mathbf{t}=(p q, \ldots, p q)$. By $f$ making informative voting efficient, $f(\mathbf{v})=p q$ if $\mathbf{v}=(p q, \ldots, p q)$.

Proof of Theorem 6. Consider a voting rule $f: \mathcal{J}^{n} \rightarrow \mathcal{J}$. First, assume $f$ is defined by (3)-(6). Clearly, $f$ is anonymous. It follows from Proposition 1 that informative voting is efficient with $f$ since for all $\mathbf{v} \in \mathcal{J}^{n}, f(\mathbf{v})=p q$ if and only if $n_{p}^{\mathbf{v}}=n_{q}^{\mathbf{v}}=n$; so, if and only if $\mathbf{v}=(p q, \ldots, p q)$. To show monotonicity of $f$, take two voting profiles $\mathbf{v}, \mathbf{v}^{\prime} \in \mathcal{J}^{n}$ such that for all $r \in f(\mathbf{v})$, the voters who vote for $r$ in $\mathbf{v}$ also vote for $r$ in $\mathbf{v}^{\prime}$.

Case 1: $f(\mathbf{v})=p q$. Then $\mathbf{v}=(p q, \ldots, p q)$. By definition, $\mathbf{v}^{\prime}=\mathbf{v}$ and $f\left(\mathbf{v}^{\prime}\right)=p q$.

Case 2: $f(\mathbf{v})=p \bar{q}$. The definition of $f$ implies either $n_{p}^{\mathbf{v}}>n_{q}^{\mathbf{v}}$ or $n_{p}^{\mathbf{v}}=n_{q}^{\mathbf{v}}<n$; and the definition of $\mathbf{v}^{\prime}$ implies $n_{p}^{\mathbf{v}^{\prime}} \geq n_{p}^{\mathbf{v}}$ and $n_{q}^{\mathbf{v}^{\prime}} \leq n_{q}^{\mathbf{v}}$. Suppose the former is true. Then, $n_{p}^{\mathbf{v}^{\prime}}>n_{q}^{\mathbf{v}^{\prime}}$ and $f\left(\mathbf{v}^{\prime}\right)=p \bar{q}$. Next, suppose $n_{p}^{\mathbf{v}}=n_{q}^{\mathbf{v}}<n$. If $\mathbf{v}^{\prime} \neq \mathbf{v}$, one has $n_{p}^{\mathbf{v}^{\prime}}>n_{p}^{\mathbf{v}}$ or $n_{q}^{\mathbf{v}^{\prime}}<n_{q}^{\mathbf{v}}$ which means $n_{p}^{\mathbf{v}^{\prime}}>n_{q}^{\mathbf{v}^{\prime}}$ and $f\left(\mathbf{v}^{\prime}\right)=p \bar{q}$. It is obvious that if $\mathbf{v}^{\prime}=\mathbf{v}$, we are done.

Case 3: $f(\mathbf{v})=\bar{p} q$. One can show that $f\left(\mathbf{v}^{\prime}\right)=\bar{p} q$ analogously to Case 2 .

It remains to show neutrality of $f$. Take two voting profiles $\mathbf{v}, \mathbf{v}^{\prime} \in \mathcal{J}^{n}$ such that $\mathbf{v}_{r}=\mathbf{v}^{\prime}{ }_{r^{\prime}}$ for every distinct $r, r^{\prime} \in\{p, q\}$ and there is no permutation of voters $\left(i_{1}, \ldots, i_{n}\right)$ with $\left(v_{i_{1}}, \ldots, v_{i_{n}}\right)=\left(v_{1}^{\prime}, \ldots, v_{n}^{\prime}\right)$. We have to show that $(*) f$ accepts $r$ in $\mathbf{v}$ if and only if $f$ accepts $r^{\prime}$ in $\mathbf{v}^{\prime}$. We distinguish 3 cases:

Case 1: $f(\mathbf{v})=p q$. It is clear that $\mathbf{v}^{\prime}=\mathbf{v}$, and $f\left(\mathbf{v}^{\prime}\right)=p q$.

Case 2: $f(\mathbf{v})=p \bar{q}$. By definition of $f$, either $n_{p}^{\mathbf{v}}>n_{q}^{\mathbf{v}}$ or $n_{p}^{\mathbf{v}}=n_{q}^{\mathbf{v}}<n$. One can see that the latter is not possible since then one could find a permutation of voters $\left(i_{1}, \ldots, i_{n}\right)$ with $\left(v_{i_{1}}, \ldots, v_{i_{n}}\right)=\left(v_{1}^{\prime}, \ldots, v_{n}^{\prime}\right)$. Suppose the former is true. By definition 
of $\mathbf{v}^{\prime}$, whenever $p(q)$ is accepted in $\mathbf{v}, q(p)$ is accepted in $\mathbf{v}^{\prime}$. This means $n_{p}^{\mathbf{v}^{\prime}}<n_{q}^{\mathbf{v}^{\prime}}$ and $f\left(\mathbf{v}^{\prime}\right)=\bar{p} q$. So, $f$ accepts $p$ in $\mathbf{v}$ and $q$ in $\mathbf{v}^{\prime}$, and it accepts $\bar{q}$ in $\mathbf{v}$ and $\bar{p}$ in $\mathbf{v}^{\prime}$. Hence, $\left(^{*}\right)$ holds.

Case 3: $f(\mathbf{v})=\bar{p} q$. One can show that $f\left(\mathbf{v}^{\prime}\right)=\bar{p} q$ analogously to Case 2 .

Conversely, let $f$ be anonymous, monotonic and neutral, and make informative voting efficient. We have to show that $\left(^{*}\right) f$ is defined by (3)-(6). By Proposition 1 and informative voting being efficient, $f(\mathbf{v})=p q$ if and only if $\mathbf{v}=(p q, \ldots, p q)$, equivalently $n_{p}^{\mathbf{v}}=n_{q}^{\mathbf{v}}=n$. Now, take a voting profile $\mathbf{v} \in \mathcal{J}^{n} \backslash\{(p q, \ldots, p q)\}$.

Case 1: $n_{p}^{\mathbf{v}}>n_{q}^{\mathbf{v}}$. Suppose for a contradiction, $f(\mathbf{v})=\bar{p} q$. Let $\mathbf{v}^{\prime}$ be a voting profile with $n_{p}^{\mathbf{v}^{\prime}}=n_{q}^{\mathbf{v}}$ and $n_{q}^{\mathbf{v}^{\prime}}=n_{p}^{\mathbf{v}}$. We start by proving the following claim.

Claim: For each combination of $k, l \in\{0, \ldots, n\}$, there is only one voting profile $\mathbf{v} \in \mathcal{J}^{n}$ with $n_{p}^{\mathbf{v}}=k$ and $n_{p}^{\mathbf{v}}=l$ up to the permutations of votes.

The claim follows from the fact that all votes containing $\bar{p}$ are $\bar{p} q$, and similarly, all votes containing $\bar{q}$ are $p \bar{q}$. Hence, subtracting number of $p(q)$ occurrences in a profile from $n$ gives the exact number of $\bar{p} q(p \bar{q})$ votes. Then, there is only one voting profile with $n_{p}^{\mathbf{v}}$ times $q$ and $n_{q}^{\mathbf{v}}$ times $p$ up to permutations of votes by the claim. Hence, by neutrality and anonymity, $f\left(\mathbf{v}^{\prime}\right)=p \bar{q}$. However, by monotonicity of $f, f\left(\mathbf{v}^{\prime}\right)=\bar{p} q$ since $n_{p}^{\mathbf{v}^{\prime}} \leq n_{p}^{\mathbf{v}}$ and $n_{q}^{\mathbf{v}^{\prime}} \geq n_{q}^{\mathbf{v}}$, a contradiction. Then, $f(\mathbf{v})=p \bar{q}$ if $n_{p}^{\mathbf{v}}>n_{q}^{\mathbf{v}}$.

Case 2: $n_{p}^{\mathbf{v}}<n_{q}^{\mathbf{v}}$. One can show that $f(\mathbf{v})=\bar{p} q$ analogously to Case 1 .

Case 3: $n_{p}^{\mathbf{v}}=n_{q}^{\mathbf{v}}<n$. By Proposition 1 and informative voting being efficient, $f(\mathbf{v}) \in\{p \bar{q}, \bar{p} q\}$.

So, $(*)$ is true.

Proof of Theorem 7. Consider a quota rule $f: \mathcal{J}^{n} \rightarrow \mathcal{J}^{*}$ with thresholds $m_{p}$ and $m_{q}$. Suppose for a contradiction, $f$ makes informative voting efficient. By Theorem 5 , this means Condition 2 holds. Moreover, $f(\mathbf{v})=p q$ if and only if $\mathbf{v}=(p q, \ldots, p q)$ by Condition 2 and informative voting being efficient. So, $m_{p}=m_{q}=n$. Now, consider any voting profile $\mathbf{v}$ which has the following property: if $n$ is even, there are $\frac{n}{2}$ times $p \bar{q}$ and $\frac{n}{2}$ times $\bar{p} q$ in $\mathbf{v}$, and if $n$ is odd, there are $\frac{n-1}{2}$ times $p \bar{q}$ and $\frac{n+1}{2}$ times $\bar{p} q$ in $\mathbf{v}$. It follows that $f(\mathbf{v})=\bar{p} \bar{q}$ since $n \geq 2$, which is not efficient for any given type profile. Hence, a contradiction. 